\title{
A 12-week, randomized, double-blind, placebo-controlled study assessing the efficacy of EGHB010, a standardized extract of Paeoniae radix and Glycyrrhizae radix, in patients with early age-related macular degeneration
}

\author{
Joo Young Kim ${ }^{1,2}$, Mirinae Kim ${ }^{1,2}$, Rae Young Kim ${ }^{1,2}$, Woo Kyung Park ${ }^{1,2}$, Young-Hoon Park ${ }^{1,2}$ \\ ${ }^{1}$ Department of Ophthalmology and Visual Science, Seoul St. Mary's Hospital, College of Medicine, The Catholic University of Korea, Seoul, \\ Korea; ${ }^{2}$ Catholic Institute for Visual Science, College of Medicine, The Catholic University of Korea, Seoul, Korea \\ Contributions: (I) Conception and design: JY Kim, M Kim, YH Park; (II) Administrative support: JY Kim, YH Park; (III) Provision of study materials \\ or patients: M Kim, RY Kim, WK Park; (IV) Collection and assembly of data: JY Kim, YH Park; (V) Data analysis and interpretation: JY Kim, M \\ Kim, YH Park; (VI) Manuscript writing: All authors; (VII) Final approval of manuscript: All authors. \\ Correspondence to: Young-Hoon Park, MD, PhD. Department of Ophthalmology and Visual Science, Seoul St. Mary's Hospital, College of Medicine, \\ The Catholic University of Korea, 222 Banpo-daero, Seocho-gu, Seoul 06591, Korea. Email: parkyh@catholic.ac.kr.
}

Background: EGHB010, a standardized extract of Paeoniae radix and Glycyrrhizae radix, inhibits choroidal neovascularization. The aim of this study is to evaluate the efficacy and safety of EGHB010 on early agerelated macular degeneration (AMD) progression inhibition.

Methods: The study was designed as a randomized, double-blind, single-center, placebo-controlled study. Subjects were 50 years of age or older, and early AMD satisfied the criteria of more than 15 small $(<63 \mu \mathrm{m})$ drusen, less than 20 intermediate $(\geq 63,<125 \mu \mathrm{m})$ drusen, or pigment abnormalities. For 12 weeks, the treatment group received EGHB010 and the control received the placebo. The main outcomes were changes in macular pigment optical density (MPOD), central macular thickness (CMT), and central choroidal thickness (CCT). Subgroup analysis was performed on subjects with MPOD $<0.75$ at baseline.

Results: Forty-eight subjects out of 94 were assigned to the treatment group, and 46 to the control group. At 12 weeks, mean MPOD of the treatment group increased by $0.04 \pm 0.27(\mathrm{P}=0.2730)$, and that of the control group decreased by $0.03 \pm 0.21(\mathrm{P}=0.7240)$, but there was no significant difference between the two groups $(\mathrm{P}=0.1234)$. There were no significant differences between the two groups in mean CMT and CCT $(\mathrm{P}=0.6718$ and 0.6608 , respectively). In subgroup analysis, there were 39 subjects with MPOD $<0.75$ in the treatment group and 36 in the control. Mean MPOD of the treatment group significantly increased by $0.09 \pm 0.25$ $(\mathrm{P}=0.0218)$, and there was a significant difference in mean MPOD at 12 weeks between the two groups $(\mathrm{P}=0.0248)$. Adverse reactions were similar in both groups, and no subjects had serious adverse events.

Conclusions: EGHB010 is expected to increase MPOD when administered to subjects with MPOD $<0.75$. EGHB010 is worth considering as a substance that inhibits the progression of early AMD.

Keywords: Age-related macular degeneration (AMD); EGHB010; Glycyrrhizae radix; macular pigment optical density (MPOD); Paeoniae radix

Submitted Jul 14, 2020. Accepted for publication Dec 18, 2020.

doi: 10.21037/atm-20-4701

View this article at: http://dx.doi.org/10.21037/atm-20-4701 


\section{Introduction}

Age-related macular degeneration (AMD) is one of the most common diseases that causes visual impairment among the elderly. The prevalence of AMD reported through the 2005-2008 National Health and Nutrition Examination Survey in the United States was 6.5\% (1). The prevalence in Korea published by the 2008-2011 Korean National Health and Nutrition Examination Survey was $6.62 \%$, of which early AMD was $6.02 \%$ and late AMD was $0.60 \%$. Among the late AMD cases, wet AMD was $0.48 \%$ and geographic atrophy (GA) was $0.12 \%$ (2).

The macula is a yellow-pigmented area at the posterior pole of the eye that is responsible for central vision and color identification (3). Macular pigment consists of two carotenoid pigments; lutein and zeaxanthin (4). These are concentrated in the macula rather than the peripheral retina (5), especially in the fovea, Henle's nerve fiber layer, and the parafovea in the inner plexiform layer $(6,7)$. When high-energy blue light $(400-500 \mathrm{~nm})$ reaches the retina, it can cause phototoxicity $(8,9)$. Moreover, the macula is susceptible to damage by oxidative stress, but these carotenoid pigments filter blue light and quench reactive oxygen species (ROS), thereby protecting the macula from light-induced oxidative damage to maintain its function (10-13). The macular pigment absorbs blue light near $480 \mathrm{~nm}$, and macular pigment optical density (MPOD) is a measure of the attenuation of blue light by macular pigments and is linearly related to the amount of lutein and zeaxanthin in the macula when integrating a region where macular pigment is deposited (6).

Early AMD is characterized by damage to the normal structure of Bruch's membrane, pigmentation in the retinal pigment epithelium (RPE), and the accumulation of extracellular deposits called drusen between the Bruch's membrane and the RPE (14). The Age-Related Eye Disease Study (AREDS) and AREDS 2 have proven that micronutrients are effective in reducing the risk of progression in the early stages of AMD $(15,16)$, but they do not completely prevent the progression of AMD. There are no known substances that can overcome the limitations of existing agents.

Wet AMD, a form of late AMD, is caused by choroidal neovascularization $(\mathrm{CNV})$, and angiogenesis factors such as vascular endothelial growth factor (VEGF) are involved in the proliferation of CNV (17). Previous studies have shown that EGHB010, a standardized extract of Paeoniae radix and Glycyrrbizae radix (ratio of 2:1), suppresses VEGF-mediated tube formation in human retinal microvascular endothelial cells (HRMECs) in vitro and retinal vascular leakage in vivo. Furthermore, it significantly inhibits CNV formation in a rat model of experimental laser-induced CNV (18), suggesting a beneficial effects in patients with wet AMD, so additional effects in early AMD were expected as well.

The aim of this study was to evaluate the effect of administering EGHB010 for 12 weeks in subjects with early AMD (Category 2) of AREDS through MPOD, spectraldomain optical coherence tomography (SD-OCT), and electroretinography (ERG) findings (15). We present the following article in accordance with the CONSORT reporting checklist (available at http://dx.doi.org/10.21037/ atm-20-4701).

\section{Methods}

\section{Study design and subjects}

The study was designed as a parallel randomized, doubleblind, single-center, placebo-controlled and was conducted at Seoul Saint Mary's Hospital. Institutional Review Board/ Ethics Committee approval was obtained (Saint Mary's Hospital, Seoul, Republic of Korea), and the study was conducted in accordance with the Declaration of Helsinki (as revised in 2013). Also, it was registered at the Clinical Research Information Service (CRIS) (registration number: KCT0005342). Each subject provided written informed consent before any study procedures were performed. A non-blind monitoring committee monitored the safety aspects of the study.

The subjects in this study were 50 years of age or older, with early AMD satisfying the criteria of AREDS Category 2 [more than 15 small $(<63 \mu \mathrm{m})$ drusen, less than 20 intermediate $(\geq 63,<125 \mu \mathrm{m})$ drusen, or no drusen if pigment abnormalities were present] (15). The study was limited to those with no significant macular abnormalities affecting visual acuity. If both eyes met the criteria, the worse side was registered.

Those with a central macular thickness (CMT) greater than $300 \mu \mathrm{m}$, higher than severe nonproliferative diabetic retinopathy, a history of infectious ocular disease, or a high risk of developing exudative AMD such as large pigment epithelial detachment, retinal hemorrhage, or GA were excluded. In addition, those with systemic diseases such as malignant tumors, renal dysfunction, liver dysfunction, electrolyte abnormalities, uncontrolled hypertension, uncontrolled diabetes, infectious diseases, 
Table 1 Composition of EGHB010 and placebo

\begin{tabular}{lll}
\hline & EGHB010 & Placebo \\
\hline Formulation & Hard brown capsule without taste and smell & \\
Daily intake & Once daily, two capsules per day (400 mg $\times 2$ capsules) & \\
Ingredients & EGHB010 (a standardized extract of Paeoniae radix and Glycyrrhizae & Dextrin $160 \mathrm{mg}$ \\
& radix) $160 \mathrm{mg}$ & \\
& Crystalline cellulose (excipient) $216.4 \mathrm{mg}$ & Crystalline cellulose (excipient) $216.4 \mathrm{mg}$ \\
& Magnesium Stearate (excipient) $12 \mathrm{mg}$ & Magnesium Stearate (excipient) $12 \mathrm{mg}$ \\
& Silicon dioxide (excipient) $7.6 \mathrm{mg}$ & Silicon dioxide (excipient) $7.6 \mathrm{mg}$ \\
& FD\&C Red No. 40 Aluminium Lake $3 \mathrm{mg}$ & FD\&C Red No.40 Aluminium Lake 3 mg \\
& FD\&C Yellow No. 5 Aluminum Lake 1 mg & FD\&C Yellow No.5 Aluminum Lake 1 mg \\
\hline
\end{tabular}

FD\&C, Federal Food, Drug, and Cosmetic Act.

and cardiopulmonary diseases and those who had taken medicines or health functional foods that might have affected this study within the last 4 weeks were excluded. Prohibited concomitant drugs were retinal (an active aldehyde form of vitamin A), lutein, zeaxanthin, omega 3, beta-carotene, astaxanthin, anthocyanin, and multivitamins.

Screening numbers were given to subjects meeting the above criteria in the order of signing the consent form at the screening visit by a blinded study coordinator, and "block randomization (block size 4)" was performed. For randomization, a statistical expert independent of this study generated a randomization number using SAS ver..9.4 (SAS Institute Inc., NC, Cary, USA), and the subjects were divided into treatment and control groups in a 1:1 ratio. Subjects themselves, researchers, and primary investigators did not know which groups the subjects belonged to. If blindness had to be broken prior to completion of the study due to an adverse event during the human application study period, the investigator contacted the randomization manager and the blindness was broken after reviewing and approving the cause. In the case of mild adverse events, medication was administered while maintaining the study progress, and the study was stopped if the subjects wanted. When the subject requests discontinuation of intervention, when it is difficult to continue the study due to a serious adverse reaction, when other serious diseases occur, when a violation of the inclusion, exclusion criteria and protocol occurs, when the subject takes a drug that is expected to affect the study, when the subject failed follow-up, it was considered as a drop-out.

Subjects in both groups consumed two capsules of the drug with water after a meal once a day. The treatment group took $320 \mathrm{mg}$ of EGHB010 per day and the control group took the same amount of the placebo for 12 weeks. EGHB010 and placebo had the same appearance. The ingredients are listed in Table 1. Evaluations were conducted through an outpatient clinic 1, 4, and 12 weeks after the start of ingestion. The study protocol is shown in Figure 1.

\section{Preparation of EGHB010}

Standardized EGHB010, produced by the method described by Ambati et al. (17), was provided by EYEGENE Co. Ltd. (Seoul, Korea). After mixing $200 \mathrm{~kg}$ of Paeoniae radix and $100 \mathrm{~kg}$ of Glycyrrbizae radix, 3,000 L of distilled water was added and extracted at $90{ }^{\circ} \mathrm{C}$ for eight hours. The extract solution was filtered and concentrated to obtain $50 \mathrm{~kg}$ of extract, which was then mixed with $120 \mathrm{~kg}$ of maltodextrin as a carrier to make an aqueous solution. The resulting mixture was spray-dried to obtain $140 \mathrm{~kg}$ of EGHB010. High-performance liquid chromatography (HPLC) analysis of paeoniflorin $(15.23 \mathrm{mg} / \mathrm{g})$ and glycyrrhizin $(3.06 \mathrm{mg} / \mathrm{g})$, which are marker compounds of EGHB010, confirmed that EGHB010 was produced to meet the manufacturing criteria. HPLC analysis of paeoniflorin and glycyrrhizin are shown in Appendix 1 and 2, and Figures S1,S2.

\section{Outcome measures}

At the first visit (screening), informed consent was obtained from the subjects and screening was performed. Height, weight, and medical history were obtained and pregnancy test was conducted. At screening, baseline (day 1), and weeks 1,4 , and 12 , slit-lamp biomicroscopy, a fundus 


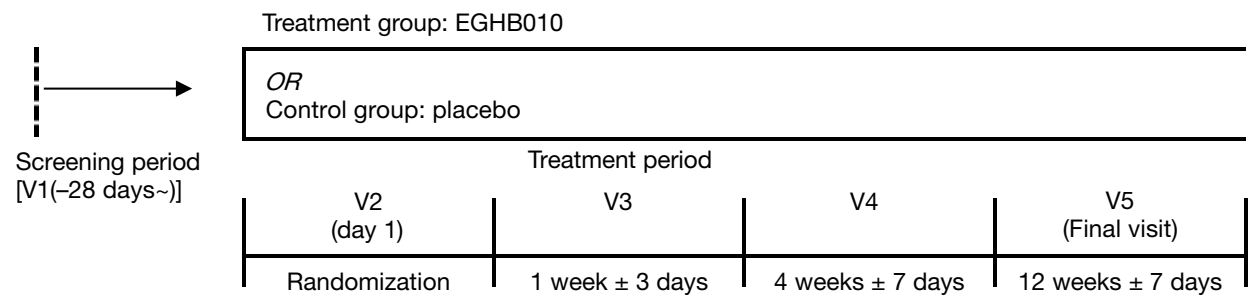

Figure 1 Study protocol.

examination, a physical examination, and an investigation of intraocular pressure, weight, vital signs, and prior and concomitant medications were performed. At screening, baseline, and weeks 4 and 12, an SD-OCT examination was performed. At screening and weeks 4 and 12, bestcorrected visual acuity and refraction were assessed, and widefield fundus photography was conducted. At weeks 1,4 , and 12 , an adverse reaction test and a compliance test were conducted. At baseline and weeks 4 and 12, the MPOD test was performed. At baseline and week 12, ERG was conducted. At screening and week 12, laboratory tests (complete blood count, blood chemistry, blood coagulation test) were conducted.

The primary outcome was the change in MPOD after 4 and 12 weeks from baseline. MPOD was measured at eccentricity of $1^{\circ}$ using a Macular Pigment Screener, MPSII $^{\circledR}$ (Elektron Technology, Cambridge, UK). The secondary outcomes were the changes in CMT and central choroidal thickness (CCT) after 4 and 12 weeks from baseline. In addition, as an indicator of visual function, the amplitude and latency of ERG 12 weeks after baseline were compared. Subgroup analysis was performed on subjects with MPOD $<0.75$ at baseline. Changes in MPOD, CMT, and CCT after 4 weeks and 12 weeks from baseline were compared between the two groups.

\section{Sample size and statistical analyses}

To satisfy the two-sample $t$-test with a power of 0.8 and a significance level of 0.05 , each group required 40 subjects. Considering the drop-out rate of about $15 \%$, the treatment group and the control group required 48 subjects, respectively, resulting in a total of 96 subjects.

An independent $t$-test or Wilcoxon's rank-sum test was used to compare two groups of continuous data. A Chisquare test was used to compare two groups of categorical data. A paired $t$-test or Wilcoxon's signed-rank test was used to analyze the differences within a group. Subgroup analysis was performed in the same way. Statistical analyses were conducted using SAS (SAS Institute Inc., NC, Cary, USA). A $P$ value of less than 0.05 was considered statistically significant.

\section{Results}

\section{Subjects flow}

Subject registration began on October 13, 2017, and the last visit of the last subject was June 28, 2019. A total of 134 subjects were screened, 38 of whom were screening failures (four violated the inclusion or exclusion criteria, 31 withdrew consent, and three for other reasons). Ninety-six subjects were randomly assigned, 48 to each group, but two in the control group voluntarily withdrew consent before the start of intervention, and the remaining 94 were defined as the full analysis set. After administration, six subjects in the treatment group and five in the control group were dropped, and the 83 subjects who completed the human application study as planned were defined as per-protocol. Of the treatment groups, three withdrew consent, and three dropped out due to cough, nasopharyngitis, and skin rash. Five out of the control group dropped out due to dyspepsia, nausea, headache, urticaria, and skin rash. An explanation of the subjects' disposition is presented in Figure 2. The demographics and baseline characteristics of subjects are summarized in Table 2.

\section{Primary and secondary outcomes}

At 12 weeks from baseline, the mean MPOD of the treatment group increased by $0.04 \pm 0.27(\mathrm{P}=0.2730)$ and that of the control group decreased by $0.03 \pm 0.21(\mathrm{P}=0.7240)$. The mean MPOD was $0.61 \pm 0.22$ in the treatment group and $0.56 \pm 0.21$ in the control group, and there was no statistically significant difference between the two groups $(\mathrm{P}=0.1234)$. Increase of $\mathrm{CMT}$ from baseline was significant 


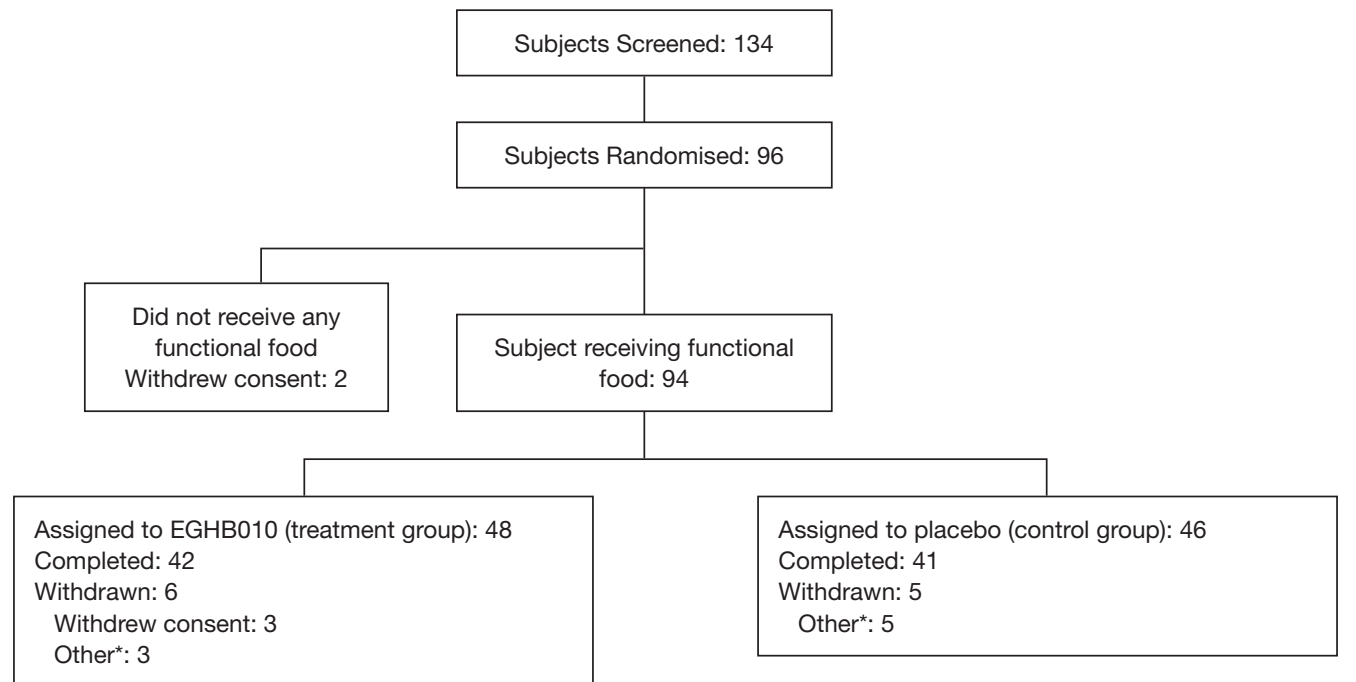

Figure 2 Subject disposition. *, three out of the treatment group dropped out due to cough, nasopharyngitis, and skin rash. In addition, five out of the control group dropped out due to dyspepsia, nausea, headache, urticaria, and skin rash.

Table 2 Demographics and baseline characteristics for treatment group and control group

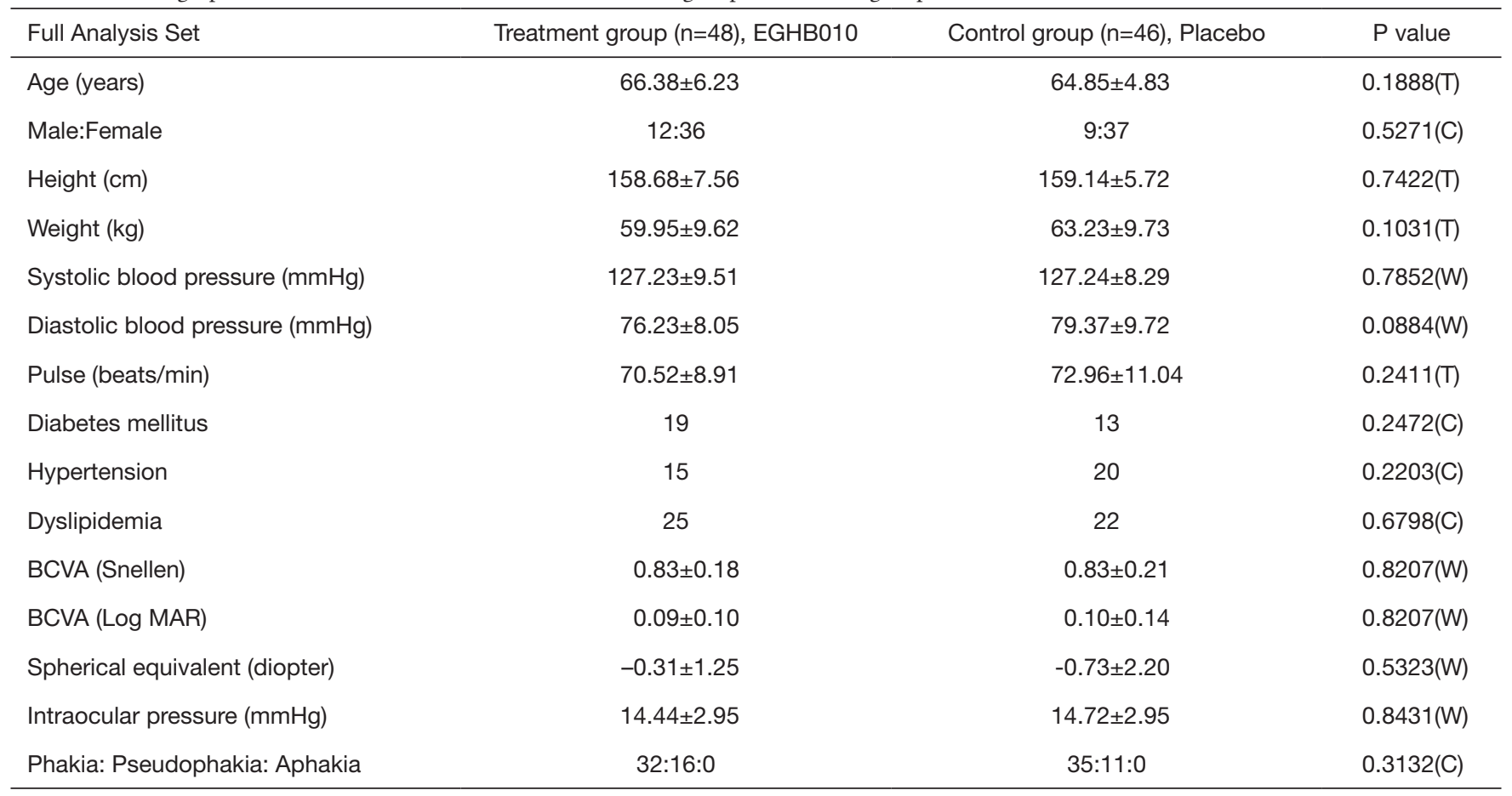

${ }^{*}$, statistically significant difference between groups $(P<0.05)$. $P$ values: independent $t$-test $(T)$, Chi-square test $(C)$, Wilcoxon's rank-sum test (W). Values are presented as mean \pm standard deviation unless otherwise indicated. BCVA, Best-corrected visual acuity.

in both groups $(\mathrm{P}<0.0001$ in the treatment group, and $\mathrm{P}=0.0033$ in the control group), but the difference between the two groups was not significant $(\mathrm{P}=0.6718)$. Although
CCT significantly increased only in the treatment group $(\mathrm{P}=0.0056)$, the difference between the two groups was not significant $(\mathrm{P}=0.6608)$. A-wave amplitude, B-wave 
amplitude, and implicit time of ERG were not significantly different from baseline at 12 weeks in both groups, and the difference between the two groups was not significant $(\mathrm{P}=0.6056,0.9849$, and 0.4042 , respectively) (Table 3).

\section{Subgroup analysis}

At baseline, there were 39 subjects with MPOD $<0.75$ in the full analysis set in the treatment group and 36 in the control group, and subgroup analysis was performed. At 12 weeks from baseline, the mean MPOD of the treatment group statistically increased by $0.09 \pm 0.25(\mathrm{P}=0.0218)$, and that of the control group increased by $0.02 \pm 0.16(\mathrm{P}=0.5605)$. The mean MPOD was $0.61 \pm 0.21$ in the treatment group and $0.52 \pm 0.17$ in the control group and significantly different $(\mathrm{P}=0.0248)$ (Table 4).

\section{Adverse reactions}

During the treatment period, mild adverse reactions occurred in 23 subjects $(47.92 \%, 34$ cases) in the treatment group and 18 (39.13\%, 27 cases) in the control group, and the difference in the incidence of adverse events between the two groups was not statistically significant $(\mathrm{P}=0.3905)$. No serious drug-related adverse reactions were noted. Adverse events were cough (16.67\%, 8 cases), nasopharyngitis ( $10.42 \%, 5$ cases), back pain $(6.25 \%, 3$ cases $)$, gastritis ( $4.17 \%, 2$ cases), headache (4.17\%, 2 cases), musculoskeletal pain $(4.17 \%, 2$ cases $)$, rash $(2.08 \%, 1$ case), oropharyngeal pain $(2.08 \%, 1$ case), nausea $(2.08 \%, 1$ case), cystitis $(2.08 \%$, 1 case), hematuria $(2.08 \%, 1$ case), dental caries $(2.08 \%, 1$ case), hemorrhoid (2.08\%, 1 case), general weakness $(2.08 \%$, 1 case), hiatal hernia (2.08\%, 1 case), colitis ( $2.08 \%, 1$ case), rotator cuff syndrome (2.08\%, 1 case), depression $(2.08 \%, 1$ case) in the treatment group. Moreover, we recorded cough ( $8.70 \%, 4$ cases), dyspepsia $(6.52 \%, 3$ cases), nasopharyngitis (6.52\%, 3 cases), rash $(6.52 \%, 3$ cases), gastritis ( $4.35 \%, 2$ cases), glare and halo ( $4.35 \%, 2$ cases), headache ( $4.35 \%$, 2 cases), influenza (2.17\%, 1 case), nausea $(2.17 \%, 1$ case), cystitis $(2.17 \%, 1$ case), dysuria $(2.17 \%, 1$ case), hematuria ( $2.17 \%, 1$ case), dental caries $(2.17 \%, 1$ case $)$, gout $(2.17 \%$, 1 case), sleep disorder $(2.17 \%, 1$ case) in the control group.

According to the vital sign results, there were no significant differences between the two groups, and none of the patients showed signs outside the normal limits. Laboratory tests, including complete blood count, blood chemistry, and blood coagulation tests, showed clinically significant increases in Aspartate Aminotransferase (AST) and Alanine Aminotransferase (ALT) (63 and 97 IU/L in one subject in the control group at 12 weeks), but it was found to be unrelated to the ingested drug. None of the other subjects showed clinically significant findings, and there were no significant differences between the two groups.

\section{Discussion}

In this study, the primary outcome, MPOD, increased in the treatment group 12 weeks after the baseline, while it decreased in the control group, but the difference between the two groups was not statistically significant. Several studies have found that the MPOD level acts as an indicator of AMD risk and that healthy people have higher MPOD than AMD patients $(6,19,20)$. The mean MPOD of aged Asian subjects measured by MPSII ${ }^{\circledR}$ was $0.73 \pm 0.16$ in the healthy group, and $0.62 \pm 0.19$ in the AMD group (21). We performed subgroup analysis on subjects with MPOD $<0.75$ except for those with high MPOD. The treatment group showed a significant increase in MPOD at 12 weeks after the baseline, and both groups had a significant difference in MPOD at the 12-week time point. This is consistent with the results of the study by Trieschmann et al. (22), in which when antioxidants were consumed, the lower the baseline MPOD, the faster the MPOD increased in response to antioxidants.

Although the macular pigment only consists of lutein and zeaxanthin, MPOD is also affected by factors otherwise unrelated to them (21). It has an inverse correlation with serum-oxidized LDL and serum triglyceride concentrations $(23,24)$ and a positive correlation with serum HDL concentration (22). It is also affected by single nucleotide polymorphisms (25) as well as by age, gender, race, UV exposure, and smoking $(22,26)$.

It has been found that the pathogenesis of AMD is related to oxidative stress (13), and antioxidant properties are among the various pharmacological activities induced by Paeoniae radix and Glycyrrbizae radix.

Paeoniae radix is the root of Paeonia lactiflora, a herbaceous perennial plant belonging to the family Paeoniaceae (27). Paeoniflorin, a monoterpene glycoside, is one of the major active components of Paeoniae radix. It has various pharmacological effects, such as being an antioxidant (28), anti-inflammatory (29), anticonvulsant (30), and muscle relaxant effects as well as preventing hyperlipidemia (31) and hyperglycemia (32). The antioxidant activity of paeoniflorin has been proven in several studies. Paeoniflorin increases 
Table 3 Comparison of the effect between treatment group and control group

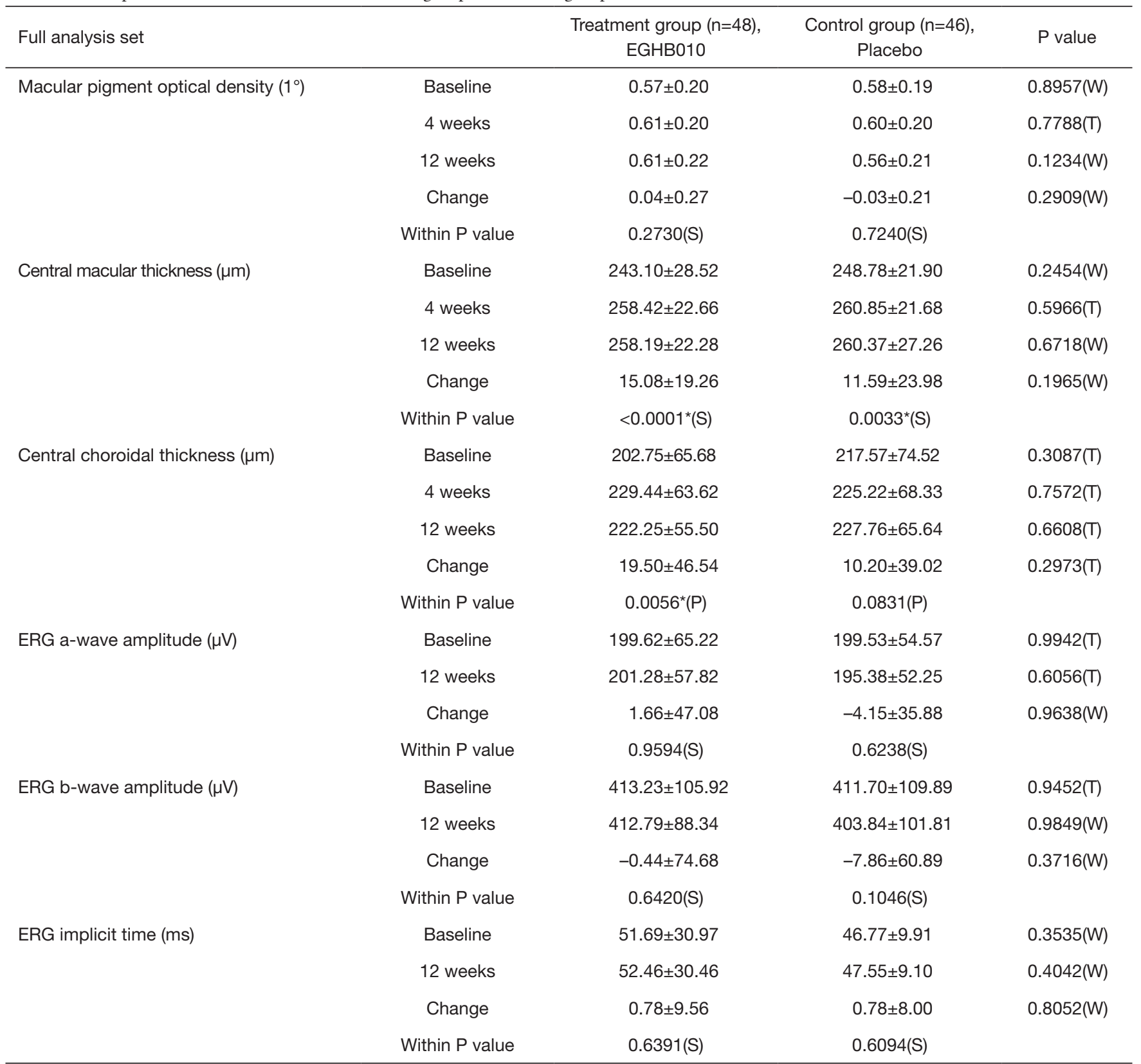

*, statistically significant difference between groups $(\mathrm{P}<0.05)$. Within $\mathrm{P}$ value: Wilcoxon's signed-rank test $(\mathrm{S})$ or $\mathrm{Paired} t$-test $(\mathrm{P})$; Between $\mathrm{P}$ value: independent $t$-test $(\mathrm{T})$ or Wilcoxon's rank-sum test $(\mathrm{W})$. Values are presented as mean \pm standard deviation unless otherwise indicated. ERG, electroretinography.

the activity of glutathione peroxidase and catalase (33), inhibits Nox $2 / \mathrm{Nox}_{4}$, a subunit of NADPH and receptor for advanced glycation end products expression, and reduces ROS production, protecting human umbilical vein endothelial cells (HUVECs) from oxidative damage (34). In the retina, paeoniflorin protects human RPE cells in vitro.
$\mathrm{H}_{2} \mathrm{O}_{2}$-induced oxidative stress increases ROS production and caspase- 3 activity, and paeoniflorin inhibits this process to protect $\mathrm{RPE}$ cells from $\mathrm{H}_{2} \mathrm{O}_{2}$-induced cell death (35). Freeform all-trans-retinal (atRAL) causes oxidative stress and mitochondrial dysfunction, leading to RPE cytotoxicity and degenerative diseases such as AMD. Recently, it was found 
Table 4 Comparison of the effect between treatment group and control group in the macular pigment optical density $<0.75$ subgroup

\begin{tabular}{|c|c|c|c|c|}
\hline Full analysis set & & $\begin{array}{l}\text { Treatment group }(n=39) \text {, } \\
\text { EGHB010 }\end{array}$ & $\begin{array}{c}\text { Control group }(\mathrm{n}=36), \\
\text { Placebo }\end{array}$ & $P$ value \\
\hline \multirow[t]{2}{*}{ Macular pigment optical density $\left(1^{\circ}\right)$} & Baseline & $0.52 \pm 0.18$ & $0.51 \pm 0.13$ & $0.4363(\mathrm{~W})$ \\
\hline & 12 weeks & $0.61 \pm 0.21$ & $0.52 \pm 0.17$ & $0.0248^{\star}(\mathrm{W})$ \\
\hline \multirow[t]{3}{*}{ Central macular thickness $(\mu \mathrm{m})$} & Baseline & $240.03 \pm 29.27$ & $244.25 \pm 21.04$ & $0.4642(\mathrm{~W})$ \\
\hline & 4 weeks & $254.79 \pm 22.65$ & $255.75 \pm 20.34$ & $0.5209(\mathrm{~W})$ \\
\hline & 12 weeks & $256.26 \pm 22.59$ & $255.19 \pm 27.41$ & $0.8903(\mathrm{~W})$ \\
\hline \multirow{4}{*}{ Central choroidal thickness $(\mu \mathrm{m})$} & 4 weeks & $235.10 \pm 59.60$ & $222.92 \pm 72.41$ & $0.4273(T)$ \\
\hline & 12 weeks & $229.28 \pm 52.63$ & $228.31 \pm 69.79$ & $0.9454(T)$ \\
\hline & Change & $20.13 \pm 48.17$ & $10.83 \pm 40.00$ & $0.3685(T)$ \\
\hline & Within $P$ value & $0.0129^{\star}(P)$ & $0.1131(\mathrm{P})$ & \\
\hline
\end{tabular}

*, statistically significant difference between groups $(\mathrm{P}<0.05)$. Within $\mathrm{P}$ value: Wilcoxon's signed-rank test $(\mathrm{S})$ or Paired $t$-test $(\mathrm{P})$; Between

$\mathrm{P}$ value: independent $t$-test (T) or Wilcoxon's rank-sum test (W).

that paeoniflorin attenuated atRAL-induced cell injury by reducing Nox1/ROS-associated oxidative stress (36). In addition, accumulation of advanced glycation end products (AGE) in drusen or RPE cells was found to be involved in CNV formation in AMD patients (37). Like EGHB010, Jakyakgamcho-tang made of Paeonia radix and Glycyrrbiza radix (especially Paeonia radix), prevents AGE formation and destroys AGE-bovine serum albumin-collagen complexes. This is thought to be due to the radical-scavenging activity of the bioactive components present in Paeonia radix (38).

Glycyrrbizae radix is obtained from the herbaceous perennial legumes Glycyrrbiza glabra and Glycyrrbiza uralensis, which belong to the family Fabaceae. Glycyrrhizin is an active component of Glycyrrbizae radix, a triterpenoid saponin, and it has been commonly used with paeoniflorin (39). It has a glucocorticoid and mineralocorticoid activity. It also anti-inflammatory, antioxidant, antiviral, and immunomodulatory effects, and improves liver function (40). Previous studies have suggested that oxidative stress is an important factor in perennial allergic rhinitis, and glycyrrhizin improves antioxidant status and reduces immune activity by reducing the incidence of free radical- induced lipid peroxidation in the blood and nasal mucosa of mice with allergic rhinitis (41). As described above, it has been found that the antioxidant activity of EGHB010 plays a positive role in AMD, but the detailed mechanism will require further research.

In this study, CMT was statistically increased in both groups, but there was no significant difference between the two groups. MPOD was not correlated with CMT in the study by Kanis et al. (42), but another study showed a positive correlation between MPOD and CMT (43). In the study by Nolan et al. (44), MPOD was not associated with CMT in Caucasian subjects, but CMT and MPOD were positively correlated in non-Caucasian subjects, suggesting that MPOD and CMT are affected by individual characteristics. In this study, there were no subjects with significantly increased CMT, and no patients complained of macular edema or decreased visual acuity.

The limitations of this study are that the 12-week period of ingestion is rather short, so that the efficacy and safety of long-term ingestion were not demonstrated. In addition, despite a variety of factors affecting MPOD, not all of them were evaluated in the subjects. 
Based on the above results, EGHB010 is expected to increase MPOD when orally administered to subjects with MPOD $<0.75$, and no serious complications were observed during the 12 -week period. This study is significant in that the clinical efficacy and safety of EGHB010 were demonstrated through a randomized controlled trial. EGHB010 is worth considering as a substance that inhibits the progression of early AMD, and further studies are needed to explain the detailed mechanisms of Paeoniae radix and Glycyrrbizae radix.

\section{Acknowledgments}

Funding: EYEGENE Inc. for the overall study and editorial assistance for this article.

\section{Footnote}

Reporting Checklist: Available at http://dx.doi.org/10.21037/ atm-20-4701

Data Sharing Statement: Available at http://dx.doi. org/10.21037/atm-20-4701

Conflicts of Interest: All authors have completed the ICMJE uniform disclosure form (available at http://dx.doi. org/10.21037/atm-20-4701). The authors have no conflicts of interest to declare.

Ethical Statement: The authors are accountable for all aspects of the work in ensuring that questions related to the accuracy or integrity of any part of the work are appropriately investigated and resolved. The study was conducted in accordance with the Declaration of Helsinki (as revised in 2013). The study was approved by Institutional Review Board/Ethics Committee of Saint Mary's Hospital, Seoul, Republic of Korea (No.: KC17HOSE0516) and informed consent was taken from all the patients. Also, it was registered at the Clinical Research Information Service (CRIS) (registration number: KCT0005342).

Open Access Statement: This is an Open Access article distributed in accordance with the Creative Commons Attribution-NonCommercial-NoDerivs 4.0 International License (CC BY-NC-ND 4.0), which permits the noncommercial replication and distribution of the article with the strict proviso that no changes or edits are made and the original work is properly cited (including links to both the formal publication through the relevant DOI and the license). See: https://creativecommons.org/licenses/by-nc-nd/4.0/.

\section{References}

1. Klein R, Chou CF, Klein BE, et al. Prevalence of agerelated macular degeneration in the US population. Arch Ophthalmol 2011;129:75-80.

2. Park SJ, Lee JH, Woo SJ, et al. Age-related macular degeneration: prevalence and risk factors from Korean National Health and Nutrition Examination Survey, 2008 through 2011. Ophthalmology 2014;121:1756-65.

3. van Lookeren Campagne M, LeCouter J, Yaspan BL, et al. Mechanisms of age-related macular degeneration and therapeutic opportunities. J Pathol 2014;232:151-64.

4. Bone RA, Landrum JT, Tarsis SL. Preliminary identification of the human macular pigment. Vision Res 1985;25:1531-5.

5. Handelman GJ, Dratz EA, Reay CC, et al. Carotenoids in the human macula and whole retina. Invest Ophthalmol Vis Sci 1988;29:850-5.

6. Bernstein PS, Delori FC, Richer S, et al. The value of measurement of macular carotenoid pigment optical densities and distributions in age-related macular degeneration and other retinal disorders. Vision Res 2010;50:716-28.

7. Trieschmann M, van Kuijk FJ, Alexander R, et al. Macular pigment in the human retina: histological evaluation of localization and distribution. Eye (Lond) 2008;22:132-7.

8. Young RW. Solar radiation and age-related macular degeneration. Surv Ophthalmol 1988;32:252-69.

9. Schalch W. Carotenoids in the retina--a review of their possible role in preventing or limiting damage caused by light and oxygen. EXS 1992;62:280-98.

10. Snodderly DM. Evidence for protection against agerelated macular degeneration by carotenoids and antioxidant vitamins. Am J Clin Nutr 1995;62:1448S-61S.

11. Sommerburg OG, Siems WG, Hurst JS, et al. Lutein and zeaxanthin are associated with photoreceptors in the human retina. Curr Eye Res 1999;19:491-5.

12. Ma L, Yan SF, Huang YM, et al. Effect of lutein and zeaxanthin on macular pigment and visual function in patients with early age-related macular degeneration. Ophthalmology 2012;119:2290-7.

13. Beatty S, Koh H, Phil M, et al. The role of oxidative stress in the pathogenesis of age-related macular degeneration. Surv Ophthalmol 2000;45:115-34.

14. Ding X, Patel M, Chan CC. Molecular pathology of 
age-related macular degeneration. Prog Retin Eye Res 2009;28:1-18.

15. Age-Related Eye Disease Study Research Group. A randomized, placebo-controlled, clinical trial of high-dose supplementation with vitamins $\mathrm{C}$ and $\mathrm{E}$, beta carotene, and zinc for age-related macular degeneration and vision loss: AREDS report no. 8. Arch Ophthalmol 2001;119:1417-36.

16. Age-Related Eye Disease Study 2 Research Group. Lutein + zeaxanthin and omega-3 fatty acids for age-related macular degeneration: the Age-Related Eye Disease Study 2 (AREDS2) randomized clinical trial. JAMA 2013;309:2005-15.

17. Ambati J, Ambati BK, Yoo SH, et al. Age-related macular degeneration: etiology, pathogenesis, and therapeutic strategies. Surv Ophthalmol 2003;48:257-93.

18. Jung E, Jung W, Park SB, et al. EGHB010, a Standardized Extract of Paeoniae Radix and Glycyrrhizae Radix, Inhibits VEGF-Induced Tube Formation In Vitro and Retinal Vascular Leakage and Choroidal Neovascularization In Vivo. Evid Based Complement Alternat Med 2017;2017:1568702.

19. Beatty S, Murray IJ, Henson DB, et al. Macular pigment and risk for age-related macular degeneration in subjects from a Northern European population. Invest Ophthalmol Vis Sci 2001;42:439-46.

20. Obana A, Hiramitsu T, Gohto Y, et al. Macular carotenoid levels of normal subjects and age-related maculopathy patients in a Japanese population. Ophthalmology 2008;115:147-57.

21. Ozawa Y, Shigeno Y, Nagai N, et al. Absolute and estimated values of macular pigment optical density in young and aged Asian participants with or without age-related macular degeneration. BMC Ophthalmol 2017;17:161.

22. Trieschmann M, Beatty S, Nolan JM, et al. Changes in macular pigment optical density and serum concentrations of its constituent carotenoids following supplemental lutein and zeaxanthin: the LUNA study. Exp Eye Res 2007;84:718-28.

23. Nagai N, Izumi-Nagai K, Suzuki M, et al. Association of macular pigment optical density with serum concentration of oxidized low-density lipoprotein in healthy adults. Retina 2015;35:820-6.

24. Loane E, Nolan JM, Beatty S. The Respective Relationships between Lipoprotein Profile, Macular Pigment Optical Density, and Serum Concentrations of Lutein and Zeaxanthin. Invest Ophthalmol Vis Sci 2010;51:5897-905.
25. McKay GJ, Loane E, Nolan JM, et al. Investigation of genetic variation in scavenger receptor class $\mathrm{B}$, member 1 (SCARB1) and association with serum carotenoids. Ophthalmology 2013;120:1632-40.

26. Hammond BR, Jr., Caruso-Avery M. Macular pigment optical density in a Southwestern sample. Invest Ophthalmol Vis Sci 2000;41:1492-7.

27. Xiang Y, Zhang Q, Wei S, et al. Paeoniflorin: a monoterpene glycoside from plants of Paeoniaceae family with diverse anticancer activities. J Pharm Pharmacol 2020;72:483-95.

28. Ryu G, Park EK, Joo JH, et al. A new antioxidant monoterpene glycoside, alpha-benzoyloxypaeoniflorin from Paeonia suffruticosa. Arch Pharm Res 2001;24:105-8.

29. Zhang W, Dai SM. Mechanisms involved in the therapeutic effects of Paeonia lactiflora Pallas in rheumatoid arthritis. Int Immunopharmacol 2012;14:27-31.

30. Abdel-Hafez AA, Meselhy MR, Nakamura N, et al. Anticonvulsant activity of paeonimetabolin-I adducts obtained by incubation of paeoniflorin and thiol compounds with Lactobacillus brevis. Biol Pharm Bull 1999;22:491-7.

31. Yang HO, Ko WK, Kim JY, et al. Paeoniflorin: an antihyperlipidemic agent from Paeonia lactiflora. Fitoterapia 2004;75:45-9.

32. Hsu FL, Lai CW, Cheng JT. Antihyperglycemic effects of paeoniflorin and 8-debenzoylpaeoniflorin, glucosides from the root of Paeonia lactiflora. Planta Med 1997;63:323-5.

33. Zhang MH, Feng L, Zhu MM, et al. Antioxidative and anti-inflammatory activities of paeoniflorin and oxypaeoniflora on AGEs-induced mesangial cell damage. Planta Med 2013;79:1319-23.

34. Song S, Xiao X, Guo D, et al. Protective effects of Paeoniflorin against AOPP-induced oxidative injury in HUVECs by blocking the ROS-HIF-1alpha/VEGF pathway. Phytomedicine 2017;34:115-26.

35. Wankun X, Wenzhen Y, Min Z, et al. Protective effect of paeoniflorin against oxidative stress in human retinal pigment epithelium in vitro. Mol Vis 2011;17:3512-22.

36. Zhu X, Wang K, Zhou F, et al. Paeoniflorin attenuates atRAL-induced oxidative stress, mitochondrial dysfunction and endoplasmic reticulum stress in retinal pigment epithelial cells via triggering $\mathrm{Ca}(2+) / \mathrm{CaMKII}$-dependent activation of AMPK. Arch Pharm Res 2018;41:1009-18.

37. Ishibashi T, Murata T, Hangai M, et al. Advanced glycation end products in age-related macular degeneration. Arch Ophthalmol 1998;116:1629-32.

38. Kim J, Kim CS, Kim YS, et al. Jakyakgamcho-tang and 
Its Major Component, Paeonia Lactiflora, Exhibit Potent Anti-glycation Properties. J Exerc Nutrition Biochem 2016;20:60-4.

39. Sun H, Wang J, Lv J. Effects of glycyrrhizin on the pharmacokinetics of paeoniflorin in rats and its potential mechanism. Pharm Biol 2019;57:550-4.

40. Dhiman RK, Chawla YK. Herbal medicines for liver diseases. Dig Dis Sci 2005;50:1807-12.

41. Li XL, Zhou AG, Zhang L, et al. Antioxidant status and immune activity of glycyrrhizin in allergic rhinitis mice.

Cite this article as: Kim JY, Kim M, Kim RY, Park WK, Park YH. A 12-week, randomized, double-blind, placebocontrolled study assessing the efficacy of EGHB010, a standardized extract of Paeoniae radix and Glycyrrbizae radix, in patients with early age-related macular degeneration. Ann Transl Med 2021;9(7):541. doi: 10.21037/atm-20-4701
Int J Mol Sci 2011;12:905-16.

42. Kanis MJ, Berendschot TT, van Norren D. Interocular agreement in melanin and macular pigment optical density. Exp Eye Res 2007;84:934-8.

43. van der Veen RL, Ostendorf S, Hendrikse F, et al. Macular pigment optical density relates to foveal thickness. Eur J Ophthalmol 2009;19:836-41.

44. Nolan JM, Stringham JM, Beatty S, et al. Spatial profile of macular pigment and its relationship to foveal architecture. Invest Ophthalmol Vis Sci 2008;49:2134-42. 


\section{Supplementary}

\section{Appendix 1 HPLC analysis of paeoniflorin in EGHB010}

1. Standard material: Paeoniflorin (Tokyo chemical industry Co. LTD, Japan)

2. Standard Working solution (in $20 \% \mathrm{MeOH}$ )

\begin{tabular}{lccccc}
\hline Standard & 1 & 2 & 3 & 4 & 5 \\
\hline Concentration $(\mu \mathrm{g} / \mathrm{mL})$ & 21.2778 & 63.8333 & 191.5000 & 383.0000 & 766.0000 \\
\hline
\end{tabular}

3. Analyzation condition

Equipment: Agilent 1260 HPLC system

Analyzation Condition

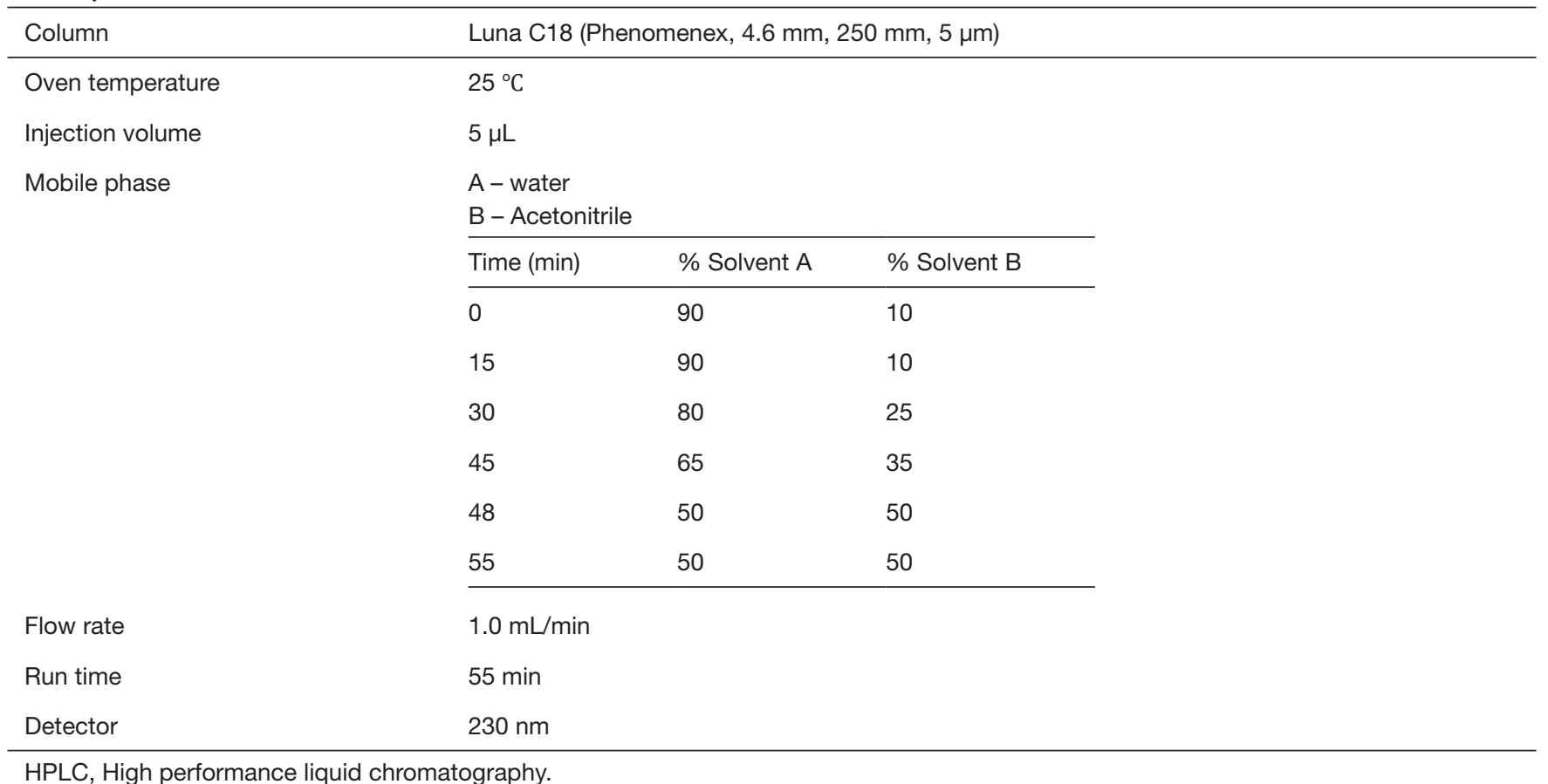




\section{Appendix 2 HPLC analysis of glycyrrhizin in EGHB010}

1. Standard material: Glycyrrhizic acid (Sigma-Aldrich, USA)

2. Standard Working solution (in $20 \% \mathrm{MeOH}$ )

\begin{tabular}{lcccccc}
\hline Standard & 1 & 2 & 3 & 4 & 5 & 6 \\
\hline Concentration $(\mu \mathrm{g} / \mathrm{mL})$ & 5.0938 & 10.1875 & 20.3750 & 40.7500 & 81.5000 & 163.0000 \\
\hline
\end{tabular}

3. Analyzation condition

Equipment: Agilent 1260 HPLC system

Analyzation Condition

\begin{tabular}{ll}
\hline Column & Luna C18 (Phenomenex, $4.6 \mathrm{~mm}, 250 \mathrm{~mm}, 5 \mu \mathrm{m})$ \\
\hline Oven temperature & $25^{\circ} \mathrm{C}$ \\
Injection volume & $10 \mu \mathrm{L}$ \\
Mobile phase & $\mathrm{A}-6.7 \%$ Acetic acid in DW \\
& $\mathrm{B}-$ Acetonitrile \\
& $\mathrm{A}: \mathrm{B}=60: 40$, isocratic \\
Flow rate & $1.0 \mathrm{~mL} / \mathrm{min}$ \\
Run time & $20 \mathrm{~min}$ \\
Detector & $254 \mathrm{~nm}$ \\
\hline
\end{tabular}

HPLC, High performance liquid chromatography. 


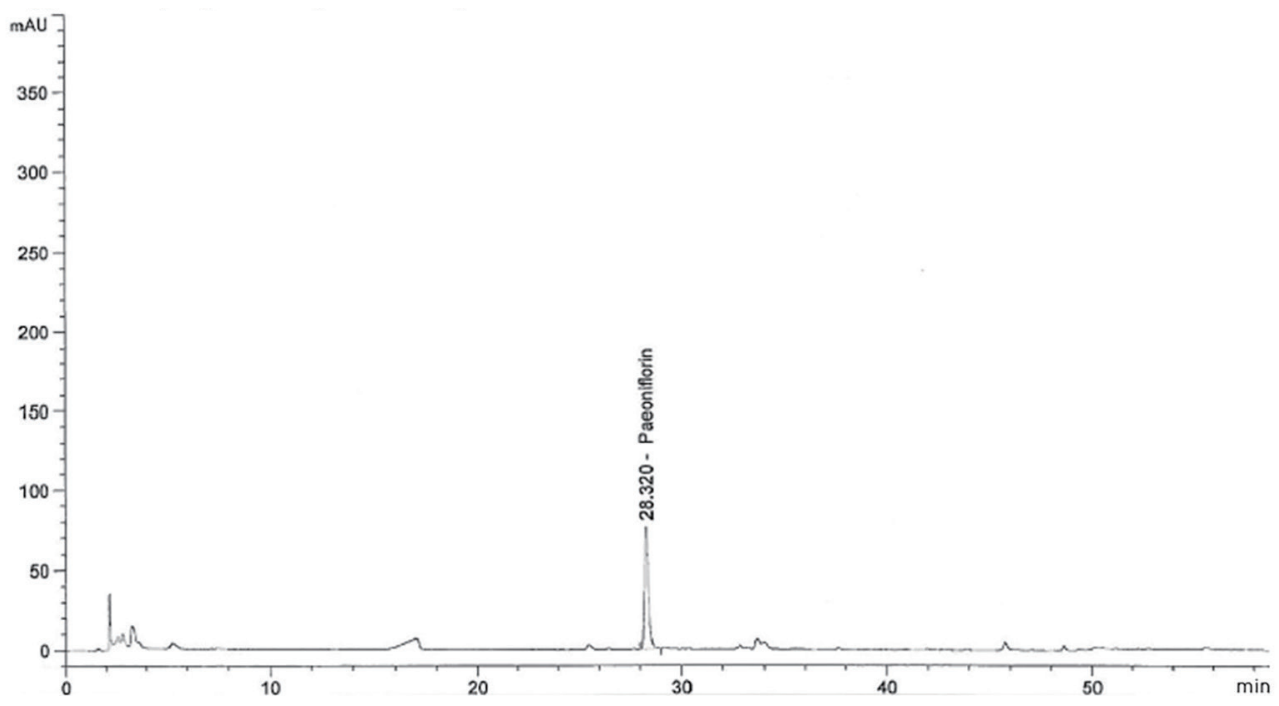

Figure S1 Representative peak of Paeoniflorin in EGHB010.

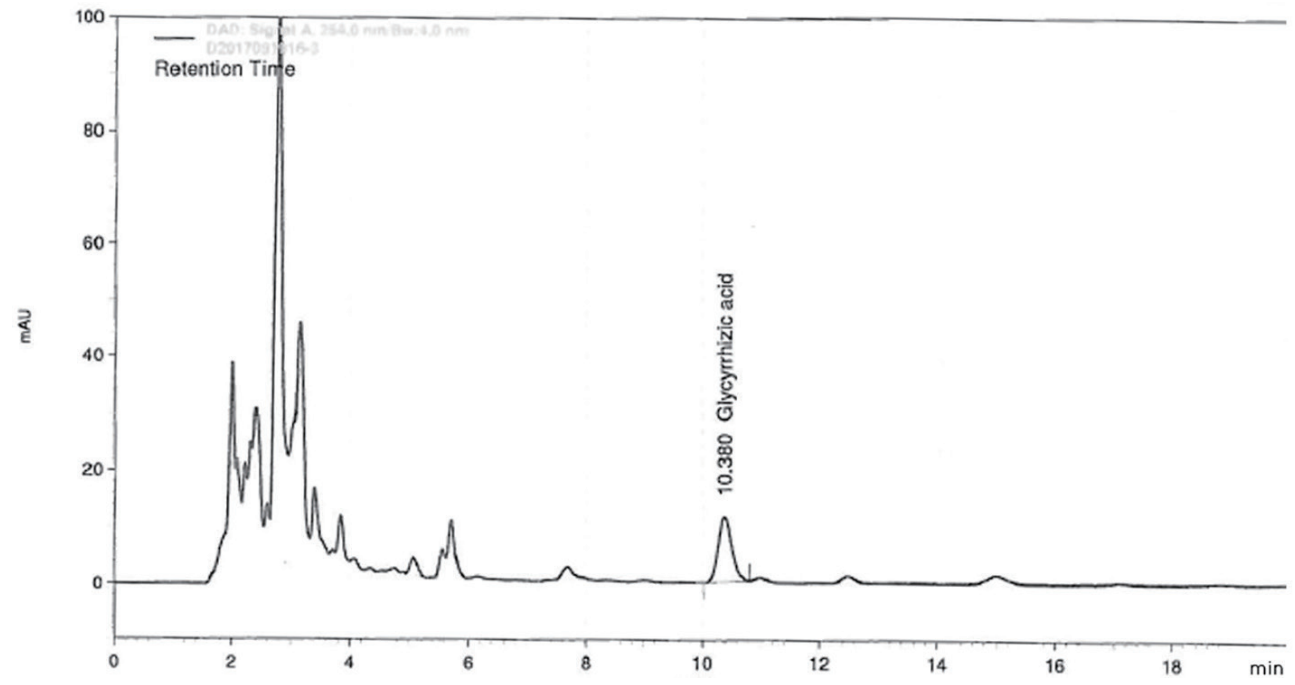

Figure S2 Representative peak of Glycyrrhizin in EGHB010. 